
\begin{abstract}
Abdulmuttalip Arslan ${ }^{6}$
1 Department of Endocrinology and Metabolism, Bolu Abant İzzet Baysal University Hospital

2 Department of Endocrinology and Metabolism, Ankara Medical Park Hospital

${ }^{3}$ Department of Endocrinology and Metabolism, Antalya Training and Educational Hospital

${ }^{4}$ Department of Endocrinology and Metabolism, Istanbul University Cerrahpasa Hospital

${ }^{5}$ Department of Endocrinology and Metabolism, Erzurum Training and Educational Hospital

${ }^{6}$ Division of Internal Medicine, Erzurum Training and Educational Hospital
\end{abstract}

Esra Ademoglu 1, Zehra Candan ${ }^{2}$, Suheyla Gorar ${ }^{3}$, Mutlu Niyazoglu 4, Ayse Carlioglu ${ }^{5}$, Faruk Yildiz ${ }^{6}$, Idris Baydar ${ }^{6}$, Hakan Sevimli ${ }^{6}$,

\title{
OBJECTIVES
}

METHODS

Acromegaly is a chronic disorder characterized by chronic growth hormone $(\mathrm{GH})$ excess. In most of cases, GH hypersecretion is derived from somatotroph cell tumors. Survivin is a member of apoptosis protein family, which was recently showed to be expressed in different benign and malignant human tumors. A number of studies showed overexpression of survivin in pituitary adenomas. This study is intended to determine circulating levels of survivin in patients with acromegaly.
The study group was composed of 19 newly diagnosed patients with acromegaly. Concurrently, 19 healthy individuals were included in the study as control group. Serum survivin levels, GH, insulin like growth factor-1 (IGF-1) and, some other biochemical parameters as fasting glucose, creatinine, alanine aminotransferase, cholesterol, triglyceride, highdensity lipoprotein cholesterol, lowdensity lipoprotein cholesterol were measured in each subject. Correlation analysis was performed between survivin and GH, IGF-1.

\section{Graphs and tables}

\begin{tabular}{|c|c|c|}
\hline & $\begin{array}{l}\text { Acromegaly } \\
(\mathrm{n}=19)\end{array}$ & $\begin{array}{l}\text { Controls } \\
(\mathrm{n}=19)\end{array}$ \\
\hline Age (year) & $40.06 \pm 11.36$ & $36.89 \pm 11.09$ \\
\hline $\mathrm{BMI}\left(\mathrm{kg} / \mathrm{m}^{2}\right)$ & $31.03 \pm 4.74$ & $28.87 \pm 7.14$ \\
\hline $\mathrm{FBG}(\mathrm{mmol} / \mathrm{L})$ & $102.42 \pm 13.9$ & $89.16 \pm 9.05$ \\
\hline Creatinin (mg/dl) & $0.81 \pm 0.22$ & $0.88 \pm 0.16$ \\
\hline $\operatorname{ALT}(\mathrm{U} / \mathrm{L})$ & $16.26 \pm 7.51$ & $24.41 \pm 17.80$ \\
\hline Total cholesterol (mmo/L) & $200.76 \pm 52.80$ & $\begin{array}{l}197.45 \pm 40.89 \\
197-1 T\end{array}$ \\
\hline Triglyceride (mol/L) & $106.52 \pm 41.89$ & $131.36 \pm 58.30$ \\
\hline $\mathrm{HDL}$ - cholesterol (mmo/l/L) & $52.60 \pm 10.70$ & $44.20 \pm 8.37$ \\
\hline \multirow{3}{*}{$\begin{array}{l}\text { LDL- cholesterol ( mmol/L) } \\
\text { Survivin (pg/mL) }\end{array}$} & $133.93 \pm 43.22$ & $115.95 \pm 50.69$ \\
\hline & $26.79 \pm 26.48$ & $20.21 \pm 23.88$ \\
\hline & $21(4.4-71.6)$ & $13(4.40-94.60)$ \\
\hline \multirow[t]{2}{*}{$\mathrm{GH}(\mathrm{ng} / \mathrm{mL})$} & $3.55 \pm 4.30$ & $1.30 \pm 2.06$ \\
\hline & $1.5(0.44-18.1)$ & $0.1(0-6.4)$ \\
\hline \multirow[t]{2}{*}{ IGF-1 (ng/mL) } & $341.91 \pm 235.26^{*}$ & $159.04 \pm 63.43^{*}$ \\
\hline & $306(6.4-750)$ & $163(79-268)$ \\
\hline
\end{tabular}

\begin{tabular}{lcc}
\multicolumn{2}{l}{ Table-2. The correlation analysis of survivin with some parameters in patients with acromegaly } \\
\hline Parameters & $\mathrm{p}$ value & r value \\
& & \\
\hline FBG & 0.67 & 0.08 \\
Insulin & 0.08 & -0.33 \\
BMI & 0.90 & 0.02 \\
Creatinin & 0.08 & 0.84 \\
ALT & 0.36 & 0.11 \\
IGF-1 & 0.90 & -0.16 \\
GH & 0.30 & \\
& \\
FBG; fasting blood glucose, BMI; body mass index, GH; growth hormone, IGF-1; insulin like growth \\
factor-1.
\end{tabular}

\section{RESULTS}

Serum survivin levels tended to be higher in acromegaly group, but this was not reach statistical significance $(p>0.05)$. Serum survivin levels were comparable among acromegaly patients and controls. Neither GH nor IGF-1 correlated with serum survivin

\section{CONCLUSIONS}

Larger scale studies are needed concerning the circulating levels of survivin in patients with acromegaly. 